\title{
Dose-volume derived nomogram as a reliable predictor of radiotherapy-induced hypothyroidism in head and neck cancer patients
}

\author{
Marin Prpic ${ }^{1,2}$, Ivan Kruljac ${ }^{3}$, Davor Kust' ${ }^{1}$, Petar Suton ${ }^{2,4}$, Neva Purgar ${ }^{1}$, Lora Kirigin Bilos ${ }^{3}$, \\ Marin Gregov', Iva Mrcela1, Maja Franceschi, ${ }^{1,2,5}$, Nikola Djakovic ${ }^{1,2}$, Ana Frobe ${ }^{1,6}$

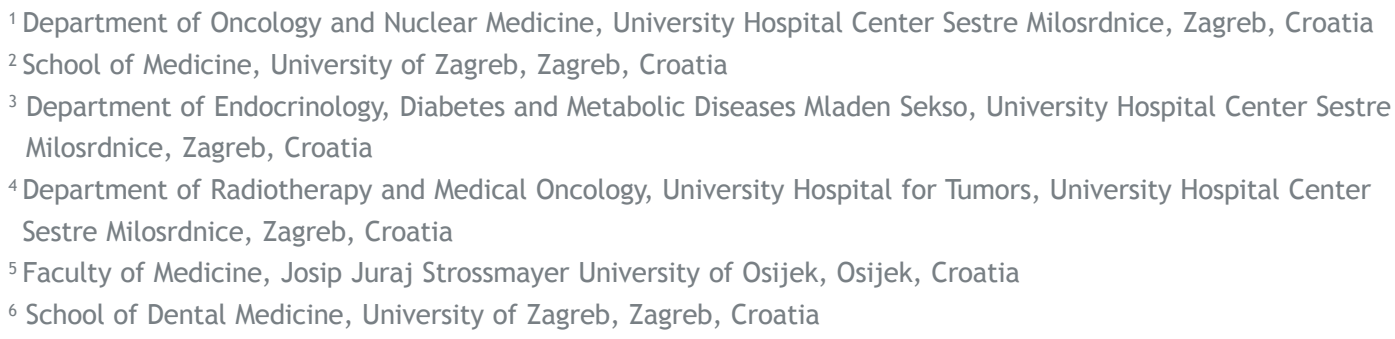

Radiol Oncol 2019; 53(4): 488-496.

Received 31 July 2019

Accepted 4 October 2019

Correspondence to: Marin Prpić, M.D., Ph.D., Department of Oncology and Nuclear Medicine, University Hospital Center Sestre Milosrdnice, Vinogradska cesta 29, 10000 Zagreb, Croatia. E-mail: marin.prpic@kbcsm.hr

Disclosure: No potential conflicts of interest were disclosed.

Background. The aim of this study was to determine the possible predictive value of various dosimetric parameters on the development of hypothyroidism (HT) in patients with head and neck squamous cell carcinoma (HNSCC) treated with (chemo)radiotherapy.

Patients and methods. This study included 156 patients with HNSCC who were treated with (chemo)radiotherapy in a primary or postoperative setting between August 2012 and September 2017. Dose-volume parameters as well as V10 toV70, D02 to D98, and the VS10 to VS70 were evaluated. The patients' hormone status was regularly assessed during follow-up. A nomogram (score) was constructed, and the Kaplan-Maier curves and Log-Rank test were used to demonstrate the difference in incidence of $\mathrm{HT}$ between cut-off values of specific variables.

Results. After a median follow-up of 23.0 (12.0-38.5) months, 70 (44.9\%) patients developed HT. In univariate analysis, VS65, Dmin, V50, and total thyroid volume (TTV) had the highest accuracy in predicting HT. In a multivariate model, HT was associated with lower TTV (OR 0.31, 95\% Cl 0.1 1-0.87, P = 0.026) and Dmin (OR 9.83, 95\% Cl 1.89-108.08, P=0.042). Hypothyroidism risk score (HRS) was constructed as a regression equation and comprised TTV and Dmin. HRS had an AUC of 0.709 (95\% Cl 0.627-0.791). HT occurred in 13 (20.0\%) patients with a score $<7.1$ and in 57 (62.6\%) patients with a score $>7.1$.

Conclusions. The dose volume parameters VS65, Dmin, V50, and TTV had the highest accuracy in predicting HT. The HRS may be a useful tool in detecting patients with high risk for radiation-induced hypothyroidism.

Key words: head and neck cancer; thyroid; radiation therapy; hypothyroidism; dose volume parameters

\section{Introduction}

A significant proportion of patients with head and neck squamous cell carcinoma (HNSCC) are treat- ed with radiation therapy at some point during their disease. Despite the use of modern techniques and advances in radiotherapy delivery, the dose at organs at risk (OARs) in the head and neck region 
is not negligible, and may result in significant side effects, such as oral mucositis, xerostomia, osteoradionecrosis, radiodermatitis and dysphagia. ${ }^{1}$ The thyroid gland, as an OAR, is frequently involved in the treatment field due to its midline neck position, and the dose it receives often exceeds $50 \mathrm{~Gy} .{ }^{2}$ Thus, one of the potential consequences of neck irradiation is development of various thyroid disorders, mainly hypothyroidism (HT), which occurs in up to $50 \%$ of patients treated with radiotherapy for HNSCC. ${ }^{3-5}$ HT is a subacute or chronic side effect of radiotherapy, with the time to development usually between 6 and 24 months after treatment. However, HT is often unpredictable and can develop at any time after completion of radiotherapy, with data showing a rising incidence with longer follow-up periods. ${ }^{6}$

Despite the growing body of evidence regarding possible damage to the thyroid during radiotherapy, there is still no generally accepted consensus on dose-volume parameters and constraints that could be used to spare the thyroid gland., 2,7-9 Moreover, the threshold dose on the thyroid gland, and the relationship between the radiation dose and induction of HT as the most important thyroid disorder resulting from radiotherapy is not well established. . $^{10-12}$

The aim of this study was to determine the possible predictive value of various dosimetric (dosevolume) parameters and clinical characteristics on the development of HT in patients with HNSCC treated with radiotherapy. Also, an additional effort was made to identify a subgroup of patients at high risk for HT development, using a combination of different factors (dose-volume nomogram).

\section{Patients and methods}

\section{Patient selection}

This study included 156 patients with HNSCC who were treated with (chemo) radiotherapy in a primary or postoperative setting between August 2012 and September 2017. Only clinically euthyroid patients were included in the study. Thyroid function was assessed during the pre-treatment diagnostic workup in all patients by evaluating the presence of thyroid-related symptoms and checking medical history, to identify possible pre-existing thyroid dysfunction. In patients with suspected HT, additional blood tests including thyroid-stimulating hormone (TSH), free thyroxine (fT4) / thyroxine (T4), and free triiodothyronine (fT3) / triiodothyronine (T3) were performed. Exclusion criteria were total thyroidectomy prior to the start of radiotherapy, patients who had irradiation to the head and neck in the past, and formerly detected thyroid disease. Additionally, patients receiving palliative radiotherapy or those with distant metastases were not included in the study, due to shorter life expectancy and thus insufficient time to develop HT. All included patients were regularly followed-up after the completion of therapy, and the study cut-off date for follow-up was March 31 2018.

The study was conducted in accordance with ethical standards set by the institutional Ethics Committee and the Helsinki Declaration from 1975, as revised in 1983. Informed consent was not needed because all patients were treated according standard protocol used in HNSCC in our department. Thyroid gland contouring during the radiotherapy planning process, and analysis of its dosimetric parameters were two additional (nonstandard) procedures, without effect on patient treatment.

\section{Treatment}

Prior to the start of radiotherapy, all patients underwent the radiation planning session (simulation) using computed tomography (CT, Toshiba Aquillion, Shimoishigami, Otawara- shi, Tochigiken, Japan), as per the institutional protocol. A thermoplastic mask (Orfit) with 5-point fixation was used for head and neck immobilization to ensure a reproducible setup. CT scan images were obtained at $2-3 \mathrm{~mm}$ slice thickness. After simulation, the data was transferred to the contouring system and the treatment planning system (Elekta Focal, $\mathrm{XiO}$ Maryland Heights, USA). Treatment was delivered using a linear accelerator (Elekta Synergy S, Elekta, Maryland Heights, USA) with 6 MV photons. Cone-beam CT was used before the first three fractions of radiotherapy and once per week afterwards for patient setup verification. Patients with adverse histopathological features (positive margins, perineural invasion, extranodal extension (ENE), multiple positive lymph nodes, stage pT3 or pT4) underwent postoperative irradiation. A dose of 46-50 Gy was given to the histologically negative and clinically undissected neck levels in daily fractions of 2 Gy. A boost of 60 Gy was applied to the tumor bed and metastases confined to the lymph node and a dose of 62-66 Gy was applied to regions of the neck with ENE and/or close/ involved margins. These latter two features were indications for the addition of chemotherapy to adjuvant irradiation (concurrent chemoradiother- 
apy). The chemotherapy regimen was cisplatin $80-$ $100 \mathrm{mg} / \mathrm{m}^{2}$ on days 1,22 , and 43 . Patient treatment planning was done using the Elekta $\mathrm{XiO}$ planning system with the three-dimensional conformal radiotherapy ConPass technique ${ }^{13}$, with an accepted planning goal of at least $95 \%$ of the planning target volume receiving more than $95 \%$ of the prescribed dose. The maximum dose was constrained to $107 \%$ of the prescribed dose. Delineated OARs were the spinal cord, parotid glands, optic nerves, retinas, eye globes, lenses, optic chiasm, cochleae, mandible and the brainstem. Additionally, the thyroid gland was contoured in all patients as an OAR, but with no specific dose constraints during treatment planning.

\section{Dosimetric analysis}

Basic thyroid gland dose-volume parameters included total thyroid volume $\left(\mathrm{cm}^{3}\right)$ - TTV, mean dose $\mathrm{D}_{\text {mean }}$ minimum $\mathrm{D}_{\min }$, and maximum $\mathrm{D}_{\max }$ dose. Furthermore, the proportion (\%) of thyroid volume receiving a dose $\mathrm{D}(\mathrm{Gy})$ in the range of doses from 10 to 70 Gy (V10 to V70, respectively), the dose to percentage (ranging from 2 to $98 \%$ ) of thyroid volume in cGy (D02 to D98, respectively), and the absolute thyroid volume spared from the dose D (Gy), again ranging from $10 \mathrm{~Gy}$ to $70 \mathrm{~Gy}$ in $\mathrm{cm}^{3}$ (VS10 to VS70, respectively) were evaluated.

\section{Clinical (non-dosimetric) parameters}

In addition to dosimetric parameters, various clinical and demographic characteristics were analysed: age, gender, localization of the primary tumor, $\mathrm{T}$ status, N status, presence of metastases, chemotherapy and surgery involving thyroid gland.

\section{Thyroid function assessment during and after HNSCC therapy}

Thyroid function was evaluated in all patients prior to the start of radiotherapy by a thyroid disease specialist who evaluated the presence of thyroidrelated symptoms and checked medical records to identify possible pre-existing hormonal imbalances. Patients with suspected thyroid dysfunction underwent further diagnostic tests to determine thyroid status. After the completion of therapy, the patients' hormone status was regularly assessed during follow-up using thyroid-stimulating hormone (TSH) and free thyroxine (fT4) assays. Thyroid hormone evaluation was done every 3 months for the first two years, and every 6 months afterwards.
Patients were evaluated using chemiluminescent microparticle immunoassay method and Abbott Architect i2000 (Abbott Diagnostics, Abbott Park, Illinois, USA). The upper limit of normal for TSH at our laboratory is $5 \mathrm{mIU} / \mathrm{L}$, so HT was defined as $\mathrm{TSH}>5 \mathrm{mIU} / \mathrm{L}$. In patients with elevated TSH and clinical symptoms of HT, thyroid hormone replacement with levothyroxine was introduced.

\section{Statistical analysis}

Patient characteristics were assessed using descriptive statistics presented as a mean with standard deviation. Continuous variables were compared with t-test or Mann-Whitney $U$ test when appropriate. Categorical variables were analysed using the Chi-squared test. Receiver operating characteristic (ROC) analysis was performed in order to determine the predictive accuracy of each variable in detecting HT. Interactions between the best predictors were analysed by using Pearson correlation analysis. The strongest predictors were then logarithmically transformed and their association with HT was analysed using the Cox proportional hazard models with a backward conditional stepwise approach. A nomogram (score) was constructed in the form of a regression equation based on unstandardized correlation coefficients derived from the final step of stepwise conditional backward Cox regression. Kaplan-Maier curves and LogRank test were used to demonstrate the difference in incidence of HT between cut-off values of specific variables. Two-sided $\mathrm{P}$ values of $<0.05$ were considered statistically significant. The statistical analysis was done using SPSS version 20.0.

\section{Results}

The mean age of the study population was $59.8 \pm$ 9.7 years and $137(87.8 \%)$ were males. After a median follow-up of $23.0(12.0-38.5)$ months at the study cut-off date, 70 (44.9\%) patients developed HT (Figure 1, Table 1). The differences in general and dosimetric parameters between patients with and without HT are presented in Tables 2 and 3.

Since no significant differences in general characteristics between patients with and without HT were found, ROC analysis was performed for all dosimetric parameters (Table 4), and the strongest predictive variable was added into the multivariate model. Overall, the best predictive factor was VS65 with an area under curve (AUC) of 0.684 (95\% confidence interval (CI) 0.600-0.768). Other predictive 
TABLE 1. The incidence of hypothyroidism in the study population

\begin{tabular}{ll}
\hline Time (months) & $\mathbf{N}(\%)$ \\
\hline 6 & $21(13.5)$ \\
12 & $37(23.7)$ \\
18 & $48(30.8)$ \\
24 & $59(37.8)$ \\
30 & $66(42.3)$ \\
36 & $70(44.9)$ \\
42 & $70(44.9)$ \\
\hline
\end{tabular}

factors were Dmin - AUC of 0.673 (95\% confidence interval (CI) $0.589-0.757$ ) and V50 -AUC of 0.630 (95\% confidence interval (CI) $0.543-0.717$ ), while total thyroid volume (TTV) was analysed separately. Thyroid volume was analysed separately because it is an independent variable and is not associated with other dosimetric parameters.

Firstly, the interaction between these mentioned four parameters was analysed. A strong correlation was found between all variables. The strongest positive correlation was found between V50 and Dmin, and between VS65 and TTV (Table 5). In order to analyse their independent association with HT, variables were logarithmically transformed, after which a multivariate backwards conditional stepwise Cox regression was employed. In a multivariate model, HT was associated with lower TTV (OR 0.312, 95\% CI 0.112-0.868, P = 0.026) and Dmin (OR 9.832, 95\% CI 1.894-108.082, P = 0.042).

Secondly, we constructed a regression equation entitled hypothyroidism risk score (HRS) comprised of TTV and Dmin:

HRS $=\log (10) \operatorname{Dmin} \times 2.286-\log (10) \mathrm{TTV} \times 1.165$

HRS substantially increased the accuracy of TTV in predicting HT (Figure 2). Log Rank $X^{2}$ of TTV was $8.73(\mathrm{P}=0.003)$ and it increased to $25.68(\mathrm{P}<$ $0.001)$ when we replaced TTV with HRS. HRS had an AUC of 0.709 (95\% CI 0.627-0.791) and a cut-off of $>7.1$ had a sensitivity of $75.7 \%$ and a specificity of $64.0 \%$. HT occurred in $13(20.0 \%)$ patients with a score $<7.1$ and in 57 (62.6\%) patients with a score $>7.1$.

Finally, we aimed to demonstrate the actual improvement in predictive accuracy when we applied HRS to a previously established predictive factor like V50. In our cohort of patients, V50 was also associated with HT as previously reported, but this association was not independent when considering other variables. V50 was capable of delineating only 14 patients with $\mathrm{V} 50<60 \%$ as those with a lower risk of HT (Figure 3A). When we employed HRS in patients with V50 $>60 \%$, additional 52 patients were categorized as those with lower risk of HT (Figure 3B).

\section{Discussion}

Severe side effects may occur in the treatment field in different organs at risk in patients with HNSCC who are receiving (chemo)radiotherapy with $\mathrm{cu}-$ rative intent. The dose-volume constraints for the thyroid gland as an OAR are a matter of debate and not clearly defined. ${ }^{10}$ Although the introduction of IMRT has marked a great advance in radiotherapy for HNSCC patients, with its ability to spare normal surrounding tissue from high radiation doses while delivering a highly conformal dose to the tumor, a significant radiation dose is still unavoidably delivered to the thyroid gland. ${ }^{2}$ Furthermore, IMRT used for locally advanced head and neck cancer may increase the radiation dose to the thyroid compared with conventional conformal radiotherapy unless appropriate thyroid dose constraints are used. ${ }^{3}$ Due

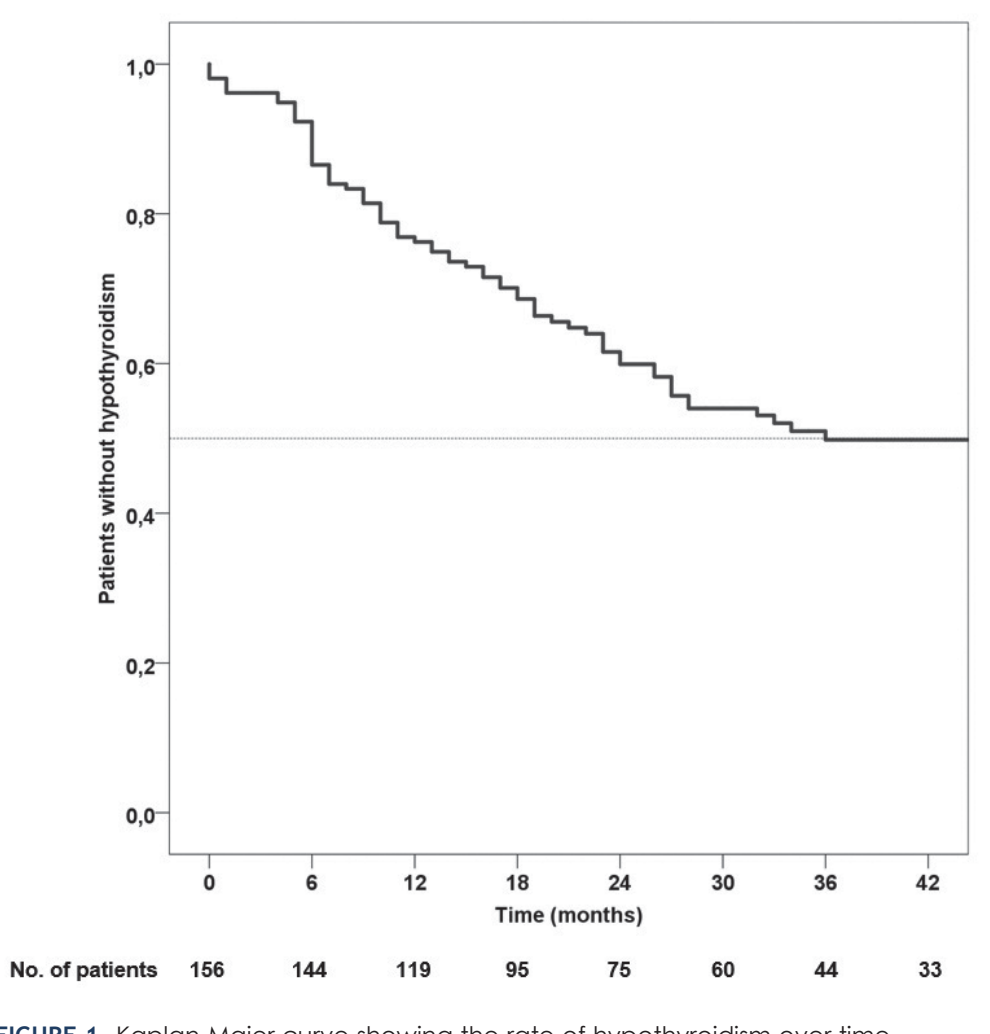

FIGURE 1. Kaplan-Maier curve showing the rate of hypothyroidism over time. 

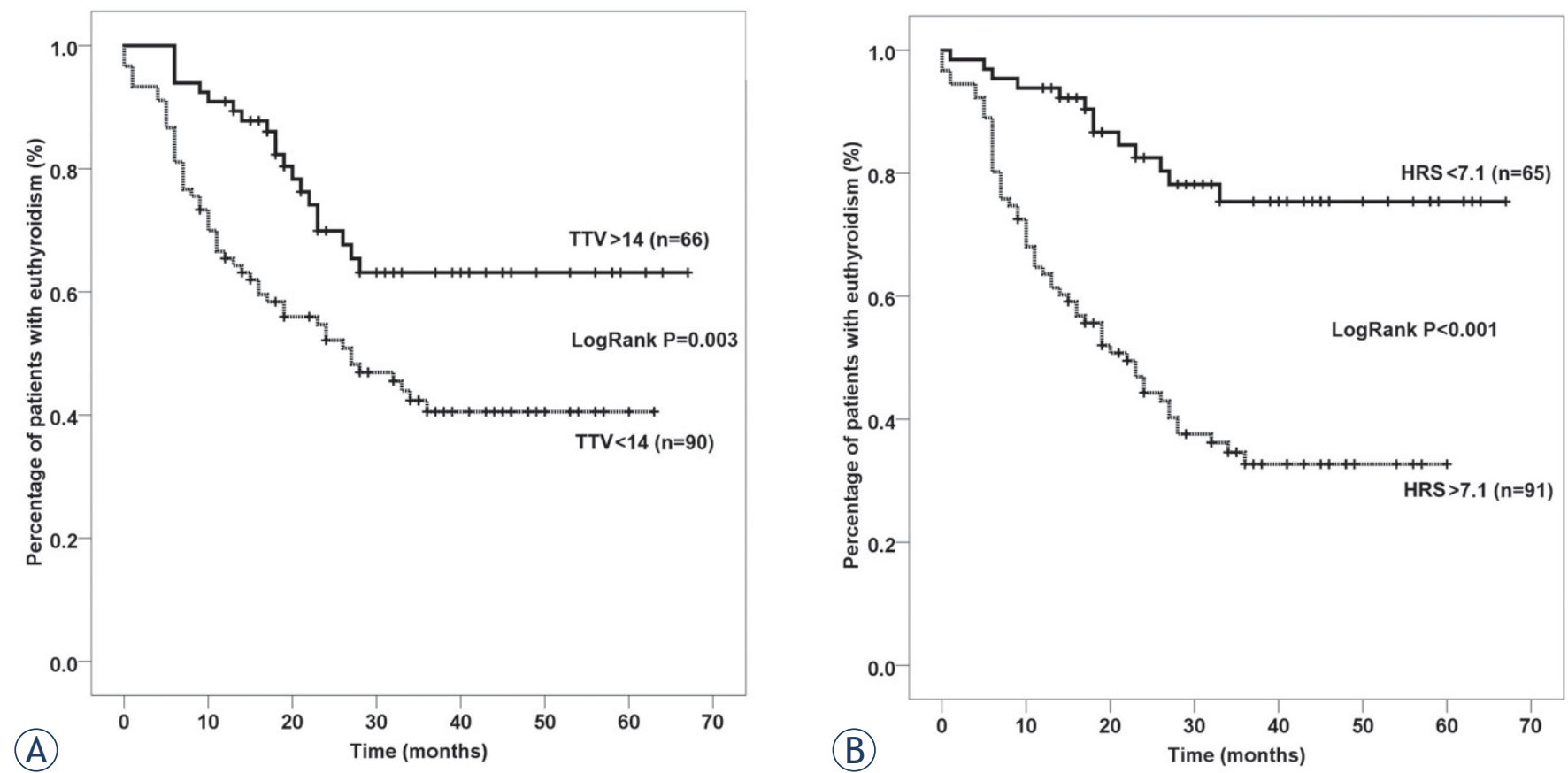

FIGURE 2. Kaplan Meier curves showing the difference in incidence of hypothyroidism between subgroups of patients divided based on specific cut-offs for total thyroid volume (TTV) (A) and Dmin (B).
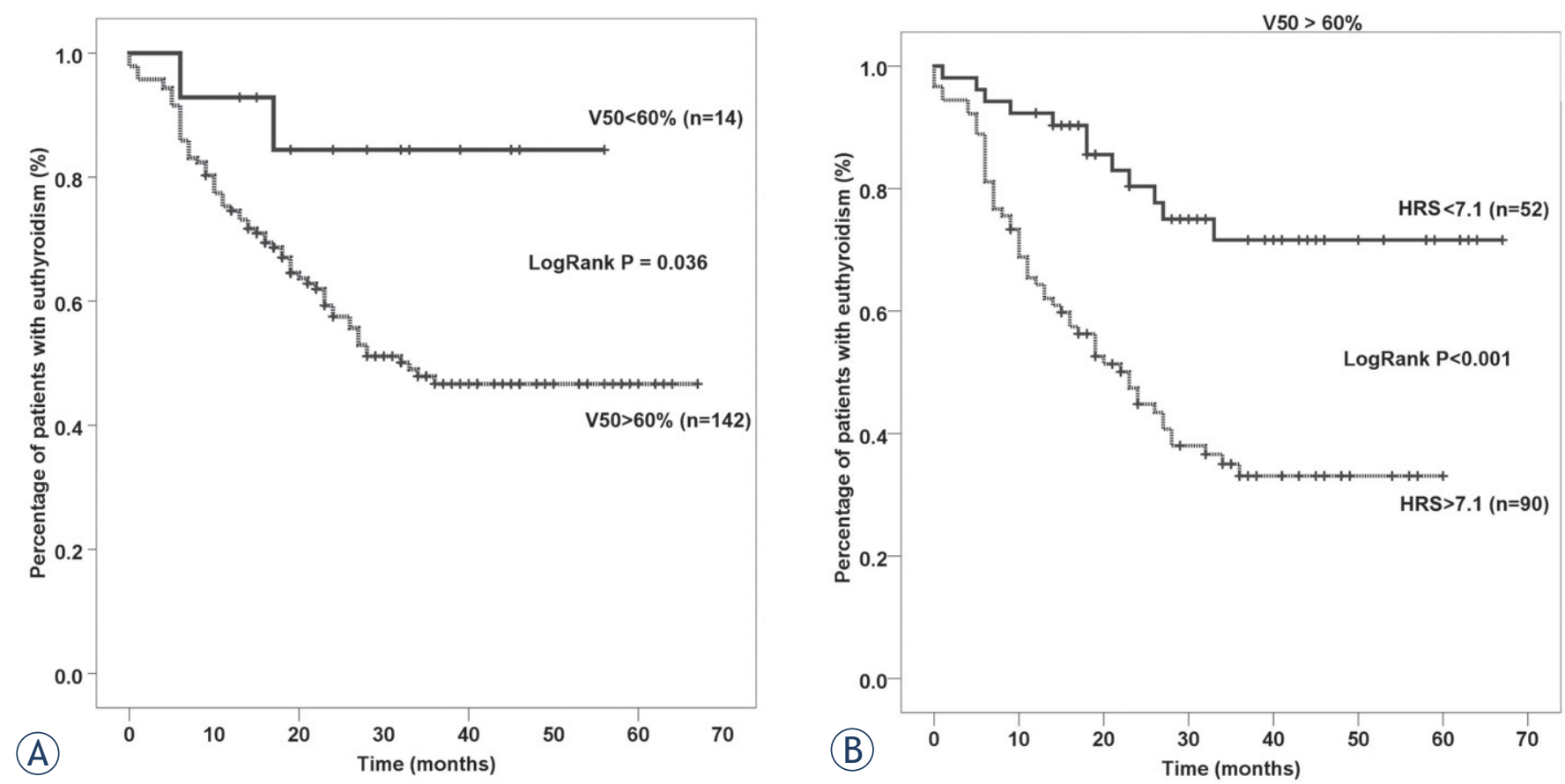

FIGURE 3. Kaplan-Meier curves showing the difference in incidence of hypothyroidism between subgroups of patients with $\mathrm{V} 50<60 \%$ and $>60 \%$ (A): Incidence of hypothyroidism in patients with $\mathrm{V} 50>60 \%$ who were subdivided based on a novel hypothyroidism risks core (HRS) comprised of Dmin and TTV (B).

to its proximity to other structures that are commonly included in the radiation field of these patients, sparing the thyroid gland can compromise final oncological outcome, especially in cases of suboptimal doses to areas which are at high-risk for recurrence. Thus, a significant proportion of patients receiving IMRT develops thyroid dysfunction, with HT being the most common complication. We observed 
a high rate of HT (44\% of patients) after a 2-yearfollow-up period, which is consistent with previous reports. $^{4-5,14}$

The relationship between the radiation dose delivered to the thyroid and the development of radiation-induced HT has been a matter of debate. Although previous studies have suggested that higher radiation doses to the thyroid gland are associated with a higher HT rate, a clear threshold radiation dose has not been defined. ${ }^{15,16}$ Recent studies on patients with Hodgkin's lymphoma, breast cancer and head and neck cancer, proposed different dose-volumetric parameters for the prediction of HT development, mostly including the proportion of thyroid volume receiving some $X$ dose (VX). In these studies, the defined VX threshold parameters ranged from V10 to V50.2,4,11,17-20 However, the proposed proportion of thyroid volume that could receive VX dose was different among studies, making it difficult to draw strong conclusions.

In the study by Kim et al. V45 was the only parameter that independently predicted HT in multivariate analysis, and V45 of 50\% was a threshold value. ${ }^{4}$ In contrast, Akgun et al. found V30 to be useful in evaluating the risk of HT, since it was a statistically significant predictor of HT development. ${ }^{17}$ In a large meta-analysis that examined dose-response data in 4 studies including a total of 1027 patients $^{10}$, final analysis showed that although there was a radiation dose-response relation with a $50 \%$ risk of HT at a dose of $45 \mathrm{~Gy}$, there was considerable variation in the dose-response between studies (the dose of $50 \%$ radiotherapy induced HT probability varied from $33 \mathrm{~Gy}$ to $65 \mathrm{~Gy}$ ), which was explained by the differences in follow-up. All authors agreed that although the threshold dose at which most patients will develop HT is still not defined, even low radiation doses have the potential to induce thyroid dysfunction. Thus, it is currently not possible to completely eliminate the risk of HT in patients treated with radiotherapy.

In the present study, V50 was found to be the best predictive factor for the development of HT among VX parameters. V50 is commonly reported in the literature as the parameter which is the most valuable in this setting; however, threshold V50 levels for the development of HT differ significantly between studies. The results of the present study are similar to those by Ling et al. which analysed radiotherapy dose parameters that corresponded with radiotherapy-induced thyroid dysfunction in 102 patients. ${ }^{8}$ Their data showed that the incidence of HT was reduced when achieving D50 < 50 Gy, V50 $<50 \%$, and a mean dose of $<54.58$ Gy. Sachdev
TABLE 2. General characteristics of the study population; continuous variables are presented as mean \pm SD and categorical ones as $\mathrm{N}(\%)$

\begin{tabular}{|c|c|c|c|c|c|}
\hline \multirow[b]{2}{*}{ Age } & \multicolumn{2}{|c|}{$\begin{array}{l}\text { Euthyroidism } \\
\mathrm{N}=86\end{array}$} & \multicolumn{2}{|c|}{$\begin{array}{l}\text { Hypothyroidism } \\
\quad \mathrm{N}=70\end{array}$} & \multirow{2}{*}{$\begin{array}{c}\mathbf{P} \\
0.556\end{array}$} \\
\hline & 60.2 & \pm 10.0 & 59.3 & \pm 9.5 & \\
\hline Male gender & 79 & (91.9) & 58 & (82.9) & 0.087 \\
\hline Primary tumor & & & & & 0.851 \\
\hline Unknown primary (C80) & 5 & (5.8) & 2 & $(2.9)$ & \\
\hline Hypopharinx & 16 & $(18.6)$ & 12 & $(17.1)$ & \\
\hline Larynx & 18 & $(20.9)$ & 16 & $(22.9)$ & \\
\hline Nasopharynx & 3 & (3.5) & 5 & $(7.1)$ & \\
\hline Oral cavity & 11 & (12.8) & 9 & (12.9) & \\
\hline Oropharynx & 28 & $(32.6)$ & 24 & (34.3) & \\
\hline Other & 5 & (5.8) & 2 & $(2.9)$ & \\
\hline T status & & & & & 0.106 \\
\hline 1 & 10 & $(11.6)$ & 14 & $(20.0)$ & \\
\hline 2 & 35 & $(40.7)$ & 22 & (31.4) & \\
\hline 3 & 16 & $(18.6)$ & 21 & $(30.0)$ & \\
\hline 4 & 17 & $(19.8)$ & 11 & $(15.7)$ & \\
\hline$x$ & 8 & (9.3) & 2 & $(2.9)$ & \\
\hline$N$ status & & & & & 0.508 \\
\hline NO & 28 & $(32.6)$ & 16 & $(22.9)$ & \\
\hline $\mathrm{Nl}$ & 16 & $(18.6)$ & 10 & (14.3) & \\
\hline N2a & 1 & $(1.2)$ & 3 & $(4.3)$ & \\
\hline $\mathrm{N} 2 \mathrm{~b}$ & 25 & $(29.1)$ & 23 & (32.9) & \\
\hline $\mathrm{N} 2 \mathrm{C}$ & 12 & $(14.0)$ & 13 & $(18.6)$ & \\
\hline N3 & 4 & $(4.7)$ & 5 & $(7.1)$ & \\
\hline Neck metastases present & 58 & $(67.4)$ & 54 & $(77.1)$ & 0.181 \\
\hline Chemotherapy & 43 & $(50.0)$ & 43 & (61.4) & 0.153 \\
\hline Surgery involving thyroid & & & & & 0.758 \\
\hline None & 18 & $(20.9)$ & 14 & $(20.0)$ & \\
\hline Lobectomy & 10 & $(11.6)$ & 11 & $(15.7)$ & \\
\hline Non-thyroid & 58 & (67.4) & 45 & (64.3) & \\
\hline
\end{tabular}

C80 = patients with unknown primary tumor, with neck metastasis present (squamous cell carcinoma), primary tumor in head and neck region was not found by sensitive diagnostics;

No surgery = no surgery of primary tumor and/of lymph nodes, chemoradiotherapy was applied: Surgery involving thyroid - none $=$ thyroid resection is not included in operation protocol Lobectomy = one lobe of the thyroid was removed

Non-thyroid = surgery of primary and/or lymph nodes was performed, without resection of the thyroid gland

et al. also reported that V50 was highly correlated with HT development, while other dosimetric parameters did not reach statistical significance. After a 50-month follow-up, the total rate of HT was $33 \%$, and the proposed threshold was V50 > $60 \% .{ }^{11}$ A V50 threshold of $<75 \%$ was proposed by Lin et al. as a useful guideline to avoid HT. ${ }^{12}$ In the paper from $\mathrm{Xu}$, the threshold level of V50 was set 
TABLE 3. Dosimetric characteristics of the study population; continuous variables are presented as mean \pm SD and categorical ones as $n(\%)$

\begin{tabular}{|c|c|c|c|c|c|}
\hline \multirow[b]{2}{*}{ Dmin } & \multicolumn{2}{|c|}{$\begin{array}{l}\text { Euthyroidism } \\
N=86\end{array}$} & \multicolumn{2}{|c|}{$\begin{array}{l}\text { Hypothyroidism } \\
N=70\end{array}$} & \multirow{2}{*}{$\begin{array}{c}\mathbf{P} \\
<0.001\end{array}$} \\
\hline & 4178 & \pm 1652 & 5005 & \pm 869 & \\
\hline Dmax & 6298 & \pm 704 & 6304 & \pm 587 & 0.867 \\
\hline Dmean & 5487 & \pm 1055 & 5836 & \pm 537 & 0.087 \\
\hline D02 & 6172 & \pm 650 & 6222 & \pm 582 & 0.765 \\
\hline D10 & 6041 & \pm 663 & 6133 & \pm 577 & 0.410 \\
\hline D20 & 5953 & \pm 709 & 6062 & \pm 567 & 0.373 \\
\hline D30 & 5818 & \pm 884 & 5992 & \pm 560 & 0.242 \\
\hline D40 & 5697 & \pm 1003 & 5927 & \pm 550 & 0.238 \\
\hline D50 & 5503 & \pm 1168 & 5869 & \pm 537 & 0.094 \\
\hline D60 & 5314 & \pm 1414 & 5775 & \pm 583 & 0.093 \\
\hline D70 & 5181 & \pm 1495 & 5704 & \pm 607 & 0.042 \\
\hline D80 & 5063 & \pm 1540 & 5616 & \pm 622 & 0.032 \\
\hline D90 & 4903 & \pm 1595 & 5520 & \pm 637 & 0.019 \\
\hline D98 & 4640 & \pm 1663 & 5322 & \pm 681 & 0.006 \\
\hline Total thyroid volume & 15.951 & \pm 8.399 & 11.461 & \pm 4.513 & $<0.001$ \\
\hline V10 & 96.85 & \pm 11.27 & 100.00 & \pm 0.00 & 0.005 \\
\hline V20 & 95.38 & \pm 14.80 & 100.00 & \pm 0.00 & 0.002 \\
\hline V30 & 94.40 & \pm 16.57 & 99.64 & \pm 2.99 & 0.005 \\
\hline V40 & 93.44 & \pm 18.36 & 99.34 & \pm 5.14 & 0.019 \\
\hline V45 & 92.70 & \pm 19.66 & 98.99 & \pm 5.51 & 0.040 \\
\hline V50 & 85.99 & \pm 24.35 & 95.29 & \pm 9.95 & 0.003 \\
\hline V55 & 53.15 & \pm 41.84 & 65.72 & \pm 40.89 & 0.053 \\
\hline V60 & 40.56 & \pm 37.74 & 49.27 & \pm 37.49 & 0.163 \\
\hline V65 & 12.82 & \pm 25.13 & 13.61 & \pm 26.67 & 0.888 \\
\hline V70 & 4.38 & \pm 15.45 & 1.81 & \pm 9.25 & 0.193 \\
\hline Vs 10 & 0.59 & \pm 2.10 & 0.00 & \pm 0.00 & 0.009 \\
\hline Vs20 & 0.87 & \pm 2.82 & 0.00 & \pm 0.00 & 0.002 \\
\hline VS30 & 1.02 & \pm 3.07 & 0.04 & \pm 0.36 & 0.008 \\
\hline VS40 & 1.23 & \pm 3.47 & 0.09 & \pm 0.72 & 0.003 \\
\hline VS45 & 1.37 & \pm 3.78 & 0.15 & \pm 0.77 & 0.040 \\
\hline VS50 & 2.56 & \pm 4.78 & 0.63 & \pm 1.45 & 0.003 \\
\hline VS55 & 7.75 & \pm 7.87 & 3.93 & \pm 5.18 & 0.003 \\
\hline VS60 & 9.86 & \pm 7.79 & 5.84 & \pm 5.16 & 0.001 \\
\hline VS65 & 14.18 & \pm 8.15 & 9.70 & \pm 4.88 & $<0.001$ \\
\hline VS70 & 15.32 & \pm 8.00 & 11.23 & \pm 4.62 & $<0.001$ \\
\hline
\end{tabular}

Dmin $=$ minimum dose $;$ Dmax = maximum dose $;$ Dmean = mean dose; D02-D98 $=$ the dose to percentage (ranging from 2 to $98 \%$ ) to thyroid volume in CGy; V10-V70 = proportion (\%) of thyroid volume receiving a dose D (Gy) in the range of doses from 10 to $70 \mathrm{~Gy}$; VS 10-VS70 = the absolute thyroid volume spared from the dose $D(G y)$ ranging from 10Gy to $70 \mathrm{~Gy}$ in $\mathrm{cm}^{3}$; $\Pi \mathrm{TV}=$ total thyroid volume $\left(\mathrm{cm}^{3}\right)$ to $54.5 \%{ }^{20}$ When all these results are analysed collectively, it can be concluded that the rate of HT is small in patients receiving < $50 \mathrm{~Gy}$. In this study, it was shown that by employing our novel scoring system (HRS) it is possible to additionally stratify a cohort of patients with V50>60\%, in order to predict the risk of HT development more precisely. Thus, this dose-volume derived nomogram could be a valuable tool in addition to the presently used parameters in everyday clinical practice.

VS is another less used dosimetric parameter which is important in estimating the amount of spared tissue. In paper by Lee et al. VS60 and VS45 of the thyroid were significant predictors of biochemical hypothyroidism. ${ }^{21}$ Freedom from biochemical hypothyroidism was longer for those whose VS60 was $\geq 10 \mathrm{~cm}^{3}$. Furthermore, in a paper by Chyan et al. VS30 Gy, VS40 Gy, and VS50 Gy were dosimetric parameters found to be statistically significant predictors of HT development. ${ }^{7}$ In the present study, VS65 was independently associated with HT in multivariate analysis, and therefore could be a useful predictive factor for radiation-induced hypothyroidism.

Data from previous studies examining the role of chemotherapy on HT development are inconclusive. Some studies have found that concurrent chemotherapy application increased the probability of HT development. ${ }^{8,19,22}$ In the study from Luo et al. chemotherapy was one factor contributing to the development of radiotherapy induced hypothyroidim, and was selected as one of the variables (risk factors), using the least absolute shrinkage and selection operator (LASSO). ${ }^{19}$ On the other hand, in the literature-based meta-analysis, which included 15 studies, chemotherapy did not affect the risk of hypothyroidism. ${ }^{10}$ However, these discrepancies may arise due to different chemotherapy doses and sequences and multi-agent chemotherapy regimens. We did not find a correlation between chemotherapy and HT, or an association between other patient and clinical characteristics (age, gender, extent of surgery, tumor site, thyroid surgery, and TNM status) and HT development. Earlier studies found that patients that underwent thyroid lobectomy had a higher incidence of HT. ${ }^{23}$ However, prior lobectomy was not found to be a predictive factor of HT in our study.

This study has several limitations including its retrospective design and relatively small number of patients. On the contrary, we believe that developing a formula that determines the risk of hypothyroidism based on the radiated thyroid volume and the dose received is of great interest. In the 
end, we would like to comment on the statistics used in this paper. Significant correlation exists between all dosimetric variables, making classical multivariate analysis impossible. We were aware of the fact that LASSO statistics was the most appropriate method to do the multivariate analysis in this case ${ }^{19}$. It might have been the most appropriate method from mathematical point of view, but it would substantially impair clarity of the results from a clinical point of view. We have performed an inferior type of LASSO statistics and decided to choose only one variable from each group which showed the strongest correlation with the onset of HT in univariate (ROC) analysis. Afterwards, we made a classical multivariate regression in order to calculate regression equation. Hence, we have made a less sophisticated variant of LASSO statistic in order to get clearer results from clinical point of view.

The nomogram presented in this paper can help in treatment decision-making, especially in HNSCC patients with a relative indication for postoperative radiotherapy. Omitting radiotherapy in some of these patients with low-risk disease (i.e. histologically negative neck, clinically negative contralateral neck in N1/N2a disease from well lateralized and small tumors) eliminates the unnecessary risk of radiation-induced side effects, including HT. In such cases, risk of radiation-induced HT predicted with the help of the nomogram (together with dosimetric constraints for other OAR) could be an important factor in treatment planning, in order to determine the best strategy for each individual patient.

The volumes and doses in head and neck radiotherapy depend mainly on the localization and extension of the local tumor as well as the levels of lymph node involvement. It is important to emphasize that sparing thyroid gland is not an option in cases when control of the tumor may be compromised. In these clinical circumstances, based on proposed nomogram, follow-up could be adjusted in those that are at highest risk for early HT development, which could lead to earlier management with hormone replacement therapy and subsequent higher quality of life.

\section{Conclusions}

In conclusion, the thyroid gland as an OAR remains a gray zone in radiotherapy. The thyroid gland is often neglected and has no priority in contouring, and because of its proximity to the tumor bed it
TABLE 4. Area under the curve for each variable in predicting hypothyroidism

\begin{tabular}{lccccc}
\hline \multirow{2}{*}{ Variable(s) } & AUC & SE & P & \multicolumn{2}{c}{$95 \%$ Confidence Interval } \\
\cline { 5 - 6 } Dmin & & & & Lower Bound & Upper Bound \\
\hline Dmax & .673 & .043 & .000 & .589 & .757 \\
\hline Dmean & .508 & .046 & .867 & .417 & .599 \\
\hline D02 & .580 & .046 & .087 & .490 & .669 \\
\hline D10 & .514 & .046 & .765 & .423 & .605 \\
\hline D20 & .538 & .046 & .410 & .448 & .629 \\
\hline D30 & .542 & .046 & .373 & .451 & .632 \\
\hline D40 & .555 & .046 & .242 & .464 & .645 \\
\hline D50 & .555 & .046 & .238 & .465 & .645 \\
\hline D60 & .578 & .046 & .094 & .489 & .668 \\
\hline D70 & .578 & .046 & .093 & .489 & .668 \\
\hline D80 & .595 & .045 & .042 & .506 & .683 \\
\hline D90 & .600 & .045 & .032 & .512 & .688 \\
\hline D98 & .609 & .045 & .019 & .521 & .697 \\
\hline VS & .627 & .044 & .006 & .540 & .714 \\
\hline
\end{tabular}

VS

$\begin{array}{llllll}\text { VS10 } & .547 & .046 & .318 & .456 & .637 \\ \text { VS20 } & .564 & .046 & .170 & .474 & .654 \\ \text { VS30 } & .557 & .046 & .218 & .468 & .647 \\ \text { VS40 } & .569 & .046 & .140 & .479 & .658 \\ \text { VS45 } & .556 & .046 & .233 & .466 & .646 \\ \text { VS50 } & .623 & .044 & .008 & .536 & .710 \\ \text { VS55 } & .637 & .044 & .003 & .550 & .723 \\ \text { VS60 } & .655 & .044 & .001 & .569 & .740 \\ \text { VS65 } & .684 & .043 & .000 & .600 & .768 \\ \text { VS70 } & .672 & .043 & .000 & .588 & .757\end{array}$

Total thyroid volume

$\begin{array}{llllll}\text { TTV } & .676 & .043 & .000 & .592 & .760\end{array}$

$\begin{array}{llllll}\text { V } & & & & & \\ \text { V10 } & .552 & .046 & .262 & .462 & .642 \\ \text { V20 } & .564 & .046 & .170 & .474 & .654 \\ \text { V30 } & .563 & .046 & .177 & .473 & .653 \\ \text { V40 } & .562 & .046 & .182 & .473 & .652 \\ \text { V45 } & .564 & .046 & .168 & .475 & .654 \\ \text { V50 } & .630 & .044 & .005 & .543 & .717 \\ \text { V55 } & .589 & .046 & .057 & .498 & .679 \\ \text { V60 } & .565 & .046 & .166 & .474 & .655 \\ \text { V65 } & .495 & .047 & .906 & .403 & .586 \\ \text { V70 } & .470 & .046 & .519 & .379 & .561\end{array}$

Dmin = minimum dose; Dmax = maximum dose; Dmean = mean dose; D02-D98 $=$ the dose to percentage (ranging from 2 to $98 \%$ ) to thyroid volume in CGy; V10-V70 = proportion (\%) of thyroid volume receiving a dose D (Gy) in the range of doses from 10 to $70 \mathrm{~Gy}$; VS 10-VS70 = the absolute thyroid volume spared from the dose $D(G y)$ ranging from $10 \mathrm{~Gy}$ to $70 \mathrm{~Gy}$ in $\mathrm{cm}^{3}$; TTV = total thyroid volume 
TABLE 5. Pearson correlation coefficients between strongest predictive variables. $\mathrm{P}<0.001$ for all correlations

\begin{tabular}{lllll}
\hline & Dmin & TTV & VS65 & V50 \\
\hline Dmin & 1.000 & -.398 & -.537 & .842 \\
TTV & -.398 & 1.000 & .853 & -.331 \\
VS65 & -.537 & .853 & 1.000 & -.462 \\
V50 & .842 & -.331 & -.462 & 1.000 \\
\hline
\end{tabular}

Dmin = minimum dose; $\operatorname{TTV}=$ total thyroid volume; $\mathrm{VS} 65=$ the absolute thyroid volume spared from the dose D (Gy) of $65 \mathrm{~Gy}$; V50 = proportion (\%) of thyroid volume receiving a dose D (Gy) of $50 \mathrm{~Gy}$

has a high exposure to radiation, especially when it is used as primary therapy. Our comprehensive analysis of dose volume parameters suggests that proposed nomogram (HRS) may be a useful tool in predicting radiation-induced hypothyroidism, which may aid in the treatment planning process. Thyroid sparing may be optimized by using V50 $<60 \%$ as a dose-volumetric threshold when possible. In cases when V50 > 60\% HRS may be helpful in predicting HT risk more precisely. Dose volume parameters should be incorporated into routine clinical practice. Although thyroid sparing should never compromise tumor coverage, hypothyroidism, as the most common manifestation of thyroid dysfunction should be anticipated and treated appropriately. These results should be tested in other studies and if validated through prospectively designed trials, could be incorporated in treatment planning and decision making in a subgroup of patients which are at low risk of recurrence when omitting radiotherapy.

\section{References}

1. Sroussi HY, Epstein JB, Bensadoun RJ, Saunders DP, Lalla RV, Migliorati CA, et al. Common oral complications of head and neck cancer radiation therapy: mucositis, infections, saliva change, fibrosis, sensory dysfunctions, dental caries, periodontal disease, and osteoradionecrosis. Cancer Med 2017; 6: 2918-31. doi: 10.1002/cam4.1221

2. Sommat K, Ong WS, Hussain A, Soong YL, Tan T, Wee J, et al. Thyroid V40 predicts primary hypothyroidism after intensity modulated radiation therapy for nasopharyngeal carcinoma. Int J Radiat Oncol Biol Phys 2017; 98: 574-80. doi: 10.1016/j.jijrobp.2017.03.007

3. Diaz R, Jaboin JJ, Morales-Paliza M, Koehler E, Phillips JG, Stinson Set, et al. Hypothyroidism as a consequence of intensity-modulated radiotherapy with concurrent taxane-based chemotherapy for locally advanced head-and-neck cancer. Int J Radiat Oncol Biol Phys 2010; 77: 468-76. doi: 10.1016/j.jirobp.2009.05.018

4. Kim MY, Yu T, Wu HG. Dose-volumetric parameters for predicting hypothyroidism after radiotherapy for head and neck cancer. Jpn J Clin Oncol 2014 44: 331-7. doi: 10.1093/jjco/hyt235

5. Mulholland GB, Zhang H, Nguyen NT, Tkacyzk N, Seikaly H, O'Connell D, et al. Optimal detection of hypothyroidism in early stage laryngeal cancer treated with radiotherapy. J Otolaryngol Head Neck Surg 2015; 44: 34. doi $10.1186 /$ s40463-015-0085-3
6. Tell R, Lundell G, Nilsson B, Sjödin H, Lewin F, Lewensohn R. Long-term incidence of hypothyroidism after radiotherapy in patients with head-andneck cancer. Int J Radiat Oncol Biol Phys 2004; 60: 395-400. doi: 10.1016/j. ijrobp.2004.03.020

7. Chyan A, Chen J, Shugard E, Lambert L, Quivey JM, Yom SS. Dosimetric predictors of hypothyroidism in oropharyngeal cancer patients treated with intensity-modulated radiation therapy. Radiat Oncol 2014; 9: 269. doi: 10.1186/s13014-014-0269-4

8. Ling $S$, Bhatt $A D$, Brown NV, Nguyen $P$, Sipos JA, Chakravarti $A$, et al. Correlative study of dose to thyroid and incidence of subsequent dysfunction after head and neck radiation. Head Neck 2017; 39: 548-54. doi: $10.1002 /$ hed.24643

9. Kanyilmaz G, Aktan M, Koc M, Demir H, Demir LS. Radiation-induced hypothyroidism in patients with breast cancer: a retrospective analysis of 243 cases. Med Dosim 2017; 42: 190-6. doi: 10.1016/j.meddos.2017.03.003

10. Vogelius IR, Bentzen SM, Maraldo MV, Petersen PM, Specht L. Risk factors for radiation-induced hypothyroidism: a literature-based meta-analysis. Cancer 2011; 117: 5250-60. doi: 10.1002/cncr.26186

11. Sachdev S, Refaat T, Bacchus ID, Sathiaseelan V, Mittal BB. Thyroid V50 highly predictive of hypothyroidism in head-and-neck cancer patients treated with intensity-modulated radiotherapy (IMRT). Am J Clin Oncol; 40: 413-7. doi: 10.1097/COC.0000000000000165

12. Lin AJ, Zhang J, Cho-Lim J, Inouye W, Lee SP. Postradiation hypothyroidism in head and neck cancers: A Department of Veterans Affairs single-institution case-control dosimetry study. Med Dosim 2018; 44: 56-60. doi: 10.1016/j. meddos.2018.02.001

13. Wiggenraad R, Mast $M$, van Santvoort J, Hoogendoorn M, Struikmans $H$ ConPas: a 3-D conformal parotidgland-sparing irradiation technique for bilateral neck treatment as an alternative to IMRT. Strahlenther Onkol 2005; 181: 673-82. doi: 10.1007/s00066-005-1413-8

14. Colevas AD, Read R, Thornhill J, Adak S, Tishler R, Busse P, et al. Hypothyroidism incidence after multimodality treatment for stage III and IV squamous cell carcinomas of the head and neck. Int J Radiat Oncol Biol Phys 2001; 51: 599-604. doi:10.1016/s0360-3016(01)01688-1

15. Boomsma MJ, Bijl HP, Langendijk JA. Radiation-induced hypothyroidism in head and neck cancer patients: a systematic review. Radiother Oncol 2011 99: 1-5. doi: 10.1016/j.radonc.2011.03.002

16. Alterio D, Jereczek-Fossa BA, Franchi B, D'Onofrio A, Piazzi V, Rondi E, et al. Thyroid disorders in patients treated with radiotherapy for head-and-neck cancer: a retrospective analysis of seventy-three patients. Int J Radiat Oncol Biol Phys 2007; 67: 144-50. doi: 10.1016/j.jirobp.2006.08.051

17. Akgun Z, Atasoy BM, Ozen Z, Yavuz D, Gulluoglu B, Sengoz M, et al. V30 as a predictor for radiation-induced hypothyroidism: a dosimetric analysis in patients who received radiotherapy to the neck. Radiat Oncol 2014; 9: 104. doi: 10.1186/1748-717X-9-104

18. Cella L, Conson M, Caterino M, De Rosa N, Liuzzi R, Picardi M, et al. Thyroid V30 predicts radiation-induced hypothyroidism in patients treated with sequential chemo-radiotherapy for Hodgkin's lymphoma. Int J Radiat Oncol Biol Phys 2012; 82: 1802-8. doi: 10.1016/j.jirobp.2010.09.054

19. Luo R, Li M, Yang Z, Zhan Y, Huang B, Lu J, et al. Nomogram for radiationinduced hypothyroidism prediction in nasopharyngeal carcinoma after treatment. Br J Radiol 2017; 90: 20160686. doi: 10.1259/bjr.20160686.

20. Xu Y, Shao Z, Tang T, Liu G, Yao Y, Wang J, et al. A dosimetric study on radiation-induced hypothyroidism following intensity-modulated radiotherapy in patients with nasopharyngeal carcinoma. Oncol Lett 2018; 16: 6126-32. doi: $10.3892 / 01.2018 .9332$

21. Lee V, Chan SY, Choi CW, Kwong D, Lam KO, Tong CC, et al. Dosimetric predictors of hypothyroidism after radical intensity-modulated radiation therapy for non-metastatic nasopharyngeal carcinoma. Clin Oncol (R Coll Radiol) 2016; 28: e52-60. doi: 10.1016/j.clon.2016.05.004

22. Luo R, Wu VWC, He B, Gao X, Xu Z, Wang D et al. Development of a normal tissue complication probability (NTCP) model for radiation-induced hypothyroidism in nasopharyngeal carcinoma patients. BMC Cancer 2018; 18: 575. doi: 10.1186/s12885-018-4348-z

23. Jereczek-Fossa BA, Alterio D, Jassem J, Gibelli B, Tradati N, Orecchia R Radiotherapy induced thyroid disorders. Cancer Treat Rev 2004; 30: 369-84. doi: 10.1016/j.ctrv.2003.12.003 